INPLASY

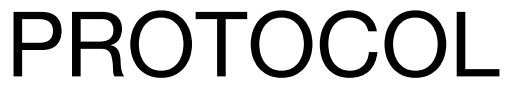

To cite: Hu et al. Different moxibustion therapies for urinary retention after anorectal surgery:A protocol for systematic review and network meta-analysis. Inplasy protocol 2020110124. doi: 10.37766/inplasy2020.11.0124

Received: 27 November 2020

Published: 27 November 2020

Corresponding author: Jinwen Hu

928081519@qq.com

Author Affiliation: Jiangxi University of Traditional Chinese Medicine

Support: None.

Review Stage at time of this submission: Preliminary searches.

Conflicts of interest: The authors declare there is no conflicts of interest regarding the publication of this paper.

\section{Different moxibustion therapies for urinary retention after anorectal surgery: A protocol for systematic review and network meta-analysis}

\author{
Hu, JW1; Hu, XY2; Sun, YP3; Shen, SL4․
}

Review question / Objective: Participants: The patients diagnosed as POUR meet the internationally recognized diagnostic criteria and have clear curative effect criteria. There are no restrictions on age, race, gender and source of cases. However, the following patients will be excluded. Interventions: The experimental group was treated with thermal moxibustion, ginger moxibustion, gentle moxibustion. Comparison: The control group was treated with conventional nursing or intramuscular injection of neostigmine. Both the experimental group and the control group could cooperate with conventional medical treatment. Outcome: The included outcome indicators included one or more of the following: 1 Effective rate: refer to "Surgery Complications" and "People's Republic of China Traditional Chinese Medicine Industry Standards.Standards for Diagnostic Efficacy of TCM Diseases", including 4 levels of recovery, markedly effective, effective and ineffective, effective rate $=[($ Healed + markedly effective + effective $) /$ total number of cases] $\times 100 \%$; 2 First urination time. 3 Residual urine volume.

INPLASY registration number: This protocol was registered with the International Platform of Registered Systematic Review and Meta-Analysis Protocols (INPLASY) on 27 November 2020 and was last updated on 27 November 2020 (registration number INPLASY2020110124).

\section{INTRODUCTION}

Review question / Objective: Participants: The patients diagnosed as POUR meet the internationally recognized diagnostic criteria and have clear curative effect criteria. There are no restrictions on age, race, gender and source of cases. However, the following patients will be excluded. Interventions: The experimental group was treated with thermal moxibustion, ginger moxibustion, gentle moxibustion. Comparison: The control 
group was treated with conventional nursing or intramuscular injection of neostigmine. Both the experimental group and the control group could cooperate with conventional medical treatment. Outcome: The included outcome indicators included one or more of the following: 1 Effective rate: refer to "Surgery Complications" and "People's Republic of China Traditional Chinese Medicine Industry Standards. Standards for Diagnostic Efficacy of TCM Diseases", including 4 levels of recovery, markedly effective, effective and ineffective, effective rate $=[($ Healed + markedly effective + effective) / total number of cases] $\times 100 \%$; 2 First urination time.3 Residual urine volume.

Condition being studied: Postoperative urinary retention (POUR) is one of the more common complications after surgery, It is also one of the common clinical emergencies at present. If it is not handled properly in time, it will not only bring great inconvenience to the life of the patient, but also affect the healing of the postoperative wound and prolong the hospitalization time. It may even cause serious consequences such as bladder rupture and kidney failure to endanger the patient Life. Now, many studies have shown that moxibustion has a significant effect on postoperative urinary retention. However, the advantages of each treatment method are different, which brings confusion to the choice of clinical operators. Therefore, this study aims to use the method of network meta analysis to treat the three methods of moxibustion (thermal moxibustion, ginger moxibustion, gentle moxibustion) in order to rank the effectiveness of urinary retention after anorectal surgery, and provide scientific evidence-based medicine basis for clinical selection.

\section{METHODS}

Participant or population: The patients diagnosed as POUR meet the internationally recognized diagnostic criteria and have clear curative effect criteria. There are no restrictions on age, race, gender and source of cases.
However, the following patients will be excluded: (1) Patients who can't tolerate moxibustion treatment; (2) Patients with severe organic diseases; (3) Pregnant women; (4) Patients with mental illness or inability to accurately describe symptoms due to unconsciousness.

Intervention: The experimental group was treated with thermal moxibustion, ginger moxibustion, gentle moxibustion. The control group was treated with conventional nursing or intramuscular injection of neostigmine. Both the experimental group and the control group could cooperate with conventional medical treatment.

Comparator: The control group was treated with conventional nursing or intramuscular injection of neostigmine. Both the experimental group and the control group could cooperate with conventional medical treatment.

Study designs to be included: Randomized controlled trials.

Eligibility criteria: Based on different moxibustion treatment of POUR RCTs, the language is limited in Chinese and English. Literature exclusion criteria: (1) Non RCTs literature, such as case report, literature review, etc; (2) Besides 3 kinds of moxibustion, other moxibustion or acupuncture therapies were used; (3) The experimental group and the control group contained other interference therapy; (4) Only one document with the most complete information was selected for the repeated detection and repeated publication; (5) Literature with incomplete data or unable to obtain data and full text; (6) Documents suspected of counterfeiting.

Information sources: Computer search Chinese databases: CNKI, Wanfang (WANFANG), VIP (VIP), Chinese Biomedical Literature Database (SinoMed), English database search PubMed, Cochrane library, Web of Science. Search terms are: "postoperative", "urinary retention", "difficult urination", "anorectal", 
"hemorrhoids", "mixed hemorrhoids", "acupuncture", "moxibustion", "thermal moxibustion","ginger moxibustion",,"gentle moxibustion".

Main outcome(s): The included outcome indicators included one or more of the following: 1 Effective rate: refer to "Surgery Complications" and "People's Republic of China Traditional Chinese Medicine Industry Standards. Standards for Diagnostic Efficacy of TCM Diseases", including 4 levels of recovery, markedly effective, effective and ineffective, effective rate $=[$ Healed + markedly effective + effective) / total number of cases] $\times 100 \%$; 2 First urination time. 3 Residual urine volume.

Quality assessment / Risk of bias analysis: According to the literature inclusion and exclusion criteria, two reviewers independently screened all relevant literature, cross-checked the screening results, and handed over to the chief Chinese physician for consultation and decision in case of disagreement. Establish a document information extraction table in Excel. The extracted information includes: title, author, publication time, number of cases, average age, gender, intervention measures, and outcome indicators.

Strategy of data synthesis: Revman $\mathbf{5 . 3}$ software was used for bias evaluation. For continuous variables (time to first urination), the results will be reported as mean difference (MD) and $95 \%$ confidence interval (Cl); count data (effective rate) will be compared by ratio (OR) and $95 \% \mathrm{Cl}$ are calculated. The outcome indicators were the ratio Ratio (or) of count data, the mean difference (MD) of measurement data, and the $95 \%$ confidence interval $(95 \% \mathrm{Cl})$ for the effect. In the heterogeneity test, if $12<$ $50 \%, P>0.10$, there is no significant heterogeneity. We chose the fixed effect model to combine the effect quantity. If the combined data is $12>50 \%, P<0.10$, it indicates high heterogeneity. We choose the random effect model to combine the effect quantity.Using Gemtc 0.14 .3 and Stata14.2 for mesh Meta analysis. In Gemtc software, Bayesian mesh Meta analysis is realized by Markov Chain Monte Carlo (MCMC) method, through 4 chains For simulation, the number of iterations is set to 50000 , and the step size is set to 10 . At the same time, the potential scale reduction parameter (Potential Scalereduced Factor, PSRF) is used to evaluate the convergence of the results. When the PSRF is close to 1 , it indicates that the results have good convergence and the obtained results are highly reliable.

Subgroup analysis: We will also conduct subgroup analysis to find more potential information.

Sensibility analysis: The purpose of sensitivity analysis is to eliminate lowquality research and explore sources of heterogeneity. Then, analyze the reliability and stability of the results by observing the heterogeneity of different studies and whether the results have changed after treatment.

\section{Country(ies) involved: China.}

Keywords: postoperative urinary retention; Anorectal; Moxibustion; Network Metaanalysis; protocol.

Contributions of each author:

Author 1 - Jinwen Hu.

Author 2 - Xiaoyang Hu.

Author 3 - Yuepeng Sun.

Author 4 - Shulan Shen. 